\title{
Interferometric technique with non-redundant sampling for phaseless inverse scattering
}

\author{
Jaime Laviada, Yuri Álvarez-López, Member, IEEE, Ana Arboleya-Arboleya, Student Member, IEEE, Cebrián \\ García-González, Student Member, IEEE, and Fernando Las-Heras, Senior Member, IEEE.
}

\begin{abstract}
A phaseless data acquisition applied on a reduced set of points is presented for inverse scattering problems. The setup is based on the Leith-Upatnieks holography with synthesized reference waves so that the phase of the reference wave can be adjusted to acquire the so-called "reduced field" instead of the regular field. Thus, non-regular sampling techniques can be applied resulting in strong reduction in the number of acquisition points. The technique is validated using simulation and measurement examples at microwave frequencies.
\end{abstract}

Index Terms-Leith-Upatnieks; indirect holography; phase retrieval; non-redundant sampling.

\section{INTRODUCTION}

I NVERSE scattering techniques are well-known to be very useful for a wide variety of applications such as medicine, defense or security. In order to lower the overall cost of the system, it is quite usual to combine them with phaseless algorithms so that the acquisition can be accomplished with low-cost power-meters.

Phaseless acquisition involves sampling requirements higher than those required for conventional amplitude and phase acquisition since the spatial bandwidth of the square amplitude is twice that of the full signal [1].

Several works have exploited the band-limited properties of the radiated fields [2] to achieve a non-redundant sampling for phaseless measurements. Thus, a complete characterization of the two-dimensional tomography has been carried out in [3], [4] for receivers and transmitters situated in multiple setups. The aforementioned techniques rely on an iterative minimization of a cost function that is free of false solutions if the setup fulfills certain geometry conditions [3], [4]. Alternatively, a one-step approach [5] has been proposed when these conditions cannot be fulfilled. However, this method is only recommended if the initial one cannot be applied due to the strong dependence on the starting guess for the iterative algorithm. Some remarkable enhancements such as the estimation of the incident field as well as extensive measurement validation are also available in the literature [6], [7].

This work has been supported by the Ministerio de Ciencia e Innovación of Spain /FEDER under projects TEC2011- 24492 (iScatt), CSD2008-00068 (Terasense) and MICINN-11-IPT-2011-0951-390000 (Tecnigraf); by the Gobierno del Principado de Asturias (PCTI)/FEDER-FSE under projects PC1006, EQUIP08-06, FC09-C0F09-12, EQUIP10-31; by Grants BP11-169, BES2009-024060.

The authors are with the Departamento de Ingeniería eléctrica, Universidad de Oviedo, Spain (e-mail: jlaviada@tsc.uniovi.es; yalopez@tsc.uniovi.es; aarboleya@tsc.uniovi.es; cgarciag@tsc.uniovi.es; flasheras@tsc.uniovi.es)
An alternative technique for phase retrieval is the LeithUpatnieks holography [8] that relies on an interferometric approach. This technique is iteration-free and, therefore, it cannot suffer from stagnation and does not require an initial guess. In addition, it has shown very good properties for the millimeter and sub-millimeter bands [9], [10] which are of special interest for the aforementioned inverse scattering applications. On the other hand, it requires a higher invest on equipment. In the case of inverse scattering problems based on monostatic scattered field, solutions have been proposed to reduce the number of extra components [11]. Moreover, a similar setup has also been proved to be able to retrieve phase information from frequency sweeps by means of processing in the time-domain [12].

A second drawback is the characterization of the reference field. This field is combined with the scattered field in the receiver and its amplitude and phase must be accurately known to avoid introducing errors into the retrieved field. Several approaches have been proposed to bypass the problem, for example, the use of synthesized reference waves [13], [14].

In the Leith-Upatnieks holography, the sampling rate also has to be increased due to the higher bandwidth of the signal resulting from combining the scattered field and the reference field [15]. In the context of antenna measurement, it has already been shown that this problem can be mitigated by properly choosing the phase of the reference wave [16]. This choice enables the acquisition of the so-called "reduced field" and, therefore, it can also benefit from the non-redundant sampling [2]. After the acquisition of the reduced field, the radiated field can be retrieved at any point by means of an optimal sampling interpolation (OSI) [17].

The main contribution of this paper is the reduction of the sampling requirements in the Leith-Upatnieks holography for inverse scattering problems based on monostatic scattered field. Thus, an efficient setup is achieved without resorting to iterative postprocessing. For this purpose, the optimum phase function to be synthesized is calculated. Furthermore, the system is experimentally validated for metallic targets by applying recent techniques to reduce the number of components [11]. The acquisition is arranged as a planar range which has been experimentally validated to be a good setup for defense and security purposes. A complete review of inverse scattering in planar ranges can be found in [18].

The paper is structured as follows. First, the setup for the implementation of the Leith-Upatnieks holography with synthetic reference wave is summarized. Next, the characterization of the quasi-monostatic field acquired by the previous 
setup is accomplished and the optimal sampling strategy is presented. After that, the optimum value for the phase of the reference field is calculated and the algorithm to retrieve the field is developed. The method is tested with several numerical examples wherein the exact results can be computed for comparison purposes. Finally, several measurements examples are shown to validate the method. The conclusions and discussion of the method end the paper.Indirect holography with nonredundant sampling

\section{A. Quasi-monostatic acquisition setup}

The Leith-Upatnieks setup for indirect holography with synthesized reference wave [13] that will be used in this paper is shown in Fig. 1. In this setup, the field scattered by the object under test (OUT) due to an incident field transmitted by an antenna is received by a second antenna, which is placed as close as possible to the transmitter antenna. The distance between both antennas is limited by the coupling between them so that it is kept under a certain threshold avoiding a significant reduction of the dynamic margin. This kind of quasi-monostatic acquisition setup can be considered monostatic from a practical point of view.

The received field is combined with a reference field that is generated from the same source of the incident field so that they are phase-locked. The most extended approach to synthesize the reference wave is to use a phase shifter in the corresponding branch. The phase shifting is adjusted so that the reference field is equivalent to the one produced by a planewave [9], [14], [19].

Although the OUT is moved along the scanning surface in this paper, same result would be obtained if the antennas were moved whereas the object remains static. For this reason, the field acquired by the aforementioned setup is characterized in the next section by assuming that the antennas are moved instead of the object.

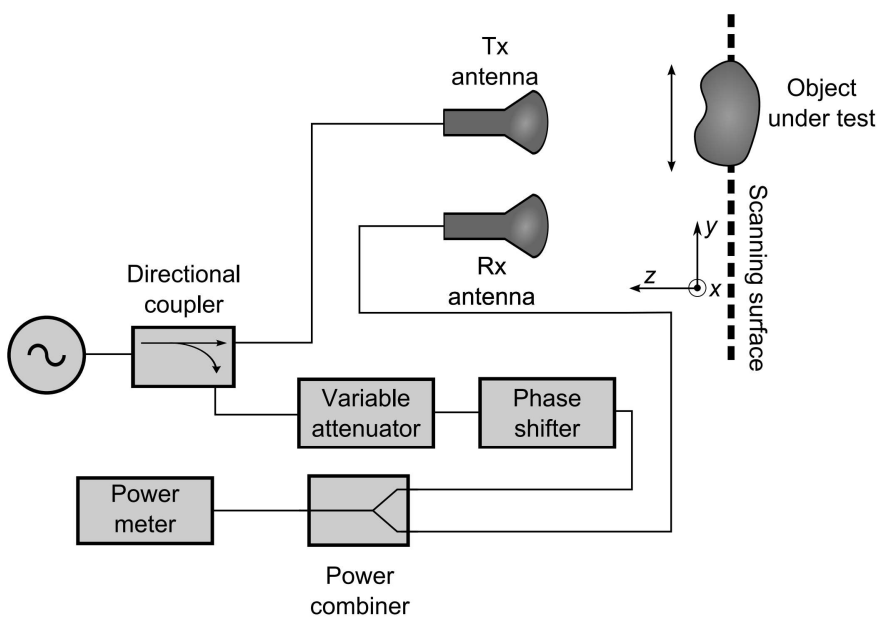

Figure 1. Implemented quasi-monostatic setup.

\section{B. Reduced monostatic field sampling}

It has been shown in [2] that an efficient representation of the field radiated by the sources enclosed on a certain convex domain $\Sigma$ on a certain curve $\mathcal{C}$ can be carried out by : i) choosing an arbitrary parametrization $\eta$ of the curve so that the observation points can be expressed as $\vec{r}=\vec{f}(\eta)$; ii) reducing the field with an arbitrary phase function $\psi$ :

$$
\vec{F}(\eta)=\vec{E}(\eta) e^{j \psi(\eta)},
$$

wherein $\vec{F}$ is the so-called reduced field. The parametrization of the observation curve as well as the phase function depend on the the shape of $\Sigma$ [2]. In the following, only the component of the field acquired by the receiver antenna will be considered and, therefore, a scalar notation is used for the field. However, the same procedure can be repeated for each component of the field.

If the parametrization and phase function, which are valid for bistatic acquisitions, are optimal [2], then the spectrum of the reduced field quickly vanishes beyond a certain value $W_{\eta}$ such that the effective spectrum of the reduced field is given by $w=\chi_{1}^{\eta} W_{\eta}$, wherein $\chi_{1}^{\eta}$ is an enlargement bandwidth factor.

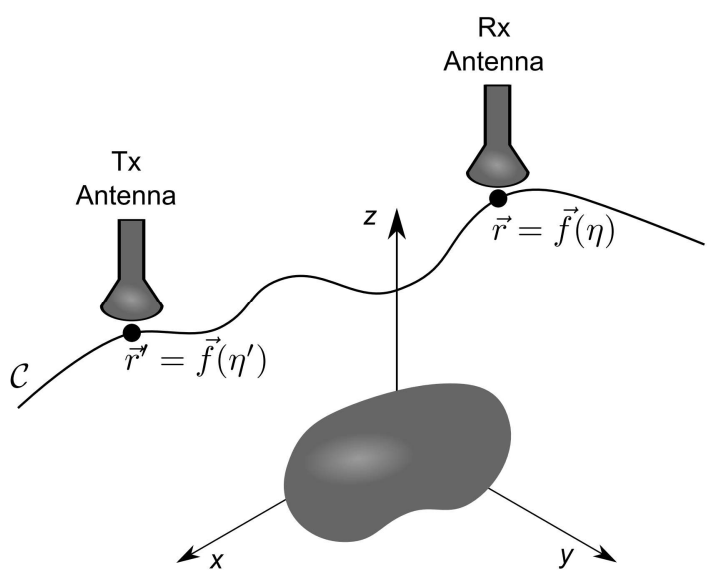

Figure 2. Field acquisition with Tx and Rx antennas at $\vec{r}^{\prime}=\vec{f}\left(\eta^{\prime}\right)$ and $\vec{r}=\vec{f}(\eta)$, respectively.

Assuming that the parametrization and phase functions are optimal, then the field scattered by a certain geometry due to the radiation of a certain antenna placed on the observation curve at a point $\eta^{\prime}$ and acquired by an antenna placed at $\eta$ (see Fig. 2) can be expanded into a Dirichlet series as [1], [2]:

$$
\begin{aligned}
& E_{s}^{(b)}\left(\eta, \eta^{\prime}\right)= \\
= & e^{-j \psi(\eta)} \sum_{u=-M^{(b)}}^{M^{(b)}} E_{s}^{(b)}\left(\eta_{u}^{(b)}, \eta^{\prime}\right) e^{j \psi\left(\eta_{u}^{(b)}\right)} D_{M^{(b)}}\left(\eta-\eta_{u}^{(b)}\right),
\end{aligned}
$$

wherein $M^{(b)}=\left\lfloor\chi_{1}^{\eta} W_{\eta}\right\rfloor+1 ; \eta_{u}^{(b)}=u \Delta \eta^{(b)}$ and $\Delta \eta^{(b)}=$ $2 \pi /\left(2 M^{(b)}+1\right)$; and $D_{M}(\cdot)$ is the periodic sinc or Dirichlet function defined as:

$$
D_{M}(x)=\frac{\sin ((2 M+1) x / 2)}{(2 M+1) \sin (x / 2)} .
$$

Please note that the superscript $b$ is used to denote parameters that are related to a bistatic acquisition.

By applying the reasoning in [1] and considering the reciprocity theorem $E_{s}^{(h)}\left(\eta_{u}^{(h)}, \eta^{\prime}\right)=E_{s}^{(h)}\left(\eta^{\prime}, \eta_{u}^{(h)}\right)$ and (2), the 
following result is achieved:

$$
\begin{aligned}
E_{s}^{(b)}\left(\eta_{u}^{(b)}, \eta^{\prime}\right)= & E_{s}^{(b)}\left(\eta^{\prime}, \eta_{u}^{(b)}\right) \\
= & e^{-j \psi\left(\eta^{\prime}\right)} \sum_{v=-M^{(b)}}^{M^{(b)}} E_{s}^{(b)}\left(\eta_{v}^{(b)}, \eta_{u}^{(b)}\right) e^{j \psi\left(\eta_{v}\right)} \\
& D_{M^{(b)}}\left(\eta^{\prime}-\eta_{v}^{(b)}\right),
\end{aligned}
$$

then, inserting (4) into (2):

$$
\begin{gathered}
E_{s}^{(b)}\left(\eta, \eta^{\prime}\right)=e^{-j \psi(\eta)} e^{-j \psi\left(\eta^{\prime}\right)} \\
\sum_{u=-M^{(b)}}^{M^{(b)}} \sum_{v=-M^{(b)}}^{M^{(b)}} E_{s}^{(b)}\left(\eta_{v}^{(b)}, \eta_{u}^{(b)}\right) e^{j \psi\left(\eta_{v}^{(b)}\right)} e^{j \psi\left(\eta_{u}^{(b)}\right)} \\
D_{M^{(b)}}\left(\eta^{\prime}-\eta_{v}\right) D_{M^{(b)}}\left(\eta-\eta_{u}^{(b)}\right) .
\end{gathered}
$$

The monostatic field $E_{s}^{(m)}(\eta)$ can be obtained from the previous one by considering $\eta=\eta^{\prime}$ in (5) yielding:

$$
\begin{gathered}
E_{s}^{(m)}(\eta)=e^{-j 2 \psi(\eta)} \sum_{u=-M^{(b)}}^{M^{(b)}} \sum_{v=-M^{(b)}}^{M^{(b)}} E_{s}^{(b)}\left(\eta_{v}^{(b)}, \eta_{u}^{(b)}\right) \\
e^{j \psi\left(\eta_{v}^{(b)}\right)} e^{j \psi\left(\eta_{u}^{(b)}\right)} D_{M^{(b)}}\left(\eta-\eta_{v}^{(b)}\right) D_{M^{(b)}}\left(\eta-\eta_{u}^{(b)}\right) .
\end{gathered}
$$

The double Dirichlet series can be turned into a single one according to [1] and, consequently:

$$
\begin{aligned}
& E_{s}^{(m)}(\eta)=e^{-j 2 \psi(\eta)} \\
& \sum_{u=-M^{(m)}}^{M^{(m)}} E_{s}^{(m)}\left(\eta_{u}^{(m)}\right) e^{j 2 \psi\left(\eta_{u}^{(m)}\right)} D_{M^{(m)}}\left(\eta-\eta_{u}^{(m)}\right),
\end{aligned}
$$

wherein $M^{(m)}=2 M^{(b)}$ and, thus, the number of samples representing the reduced monostatic field is twice the number of samples representing the reduced bistatic field. Hence, the new sampling points are given by $\eta_{u}^{(m)}=u \Delta \eta^{(m)}=$ $2 \pi u /\left(4 M^{(b)}+1\right)$ and, therefore, the spatial bandwidth of the reduced monostatic field $F_{s}^{(m)}=E_{s}^{(m)}(\eta) e^{j 2 \psi(\eta)}$ is approximately twice the bandwidth of the bistatic scattered field. Furthermore, the monostatic phase factor for phase reduction has been shown to be twice the conventional factor for bistatic acquisitions [2].

In practice, interpolation in (7) is restricted to the nearest $2 p$ samples [2], [16] to speed up the interpolation procedure. In order to minimize the error due to this truncation, an oversampling factor $\chi_{2}$ is introduced so that the sampling step is calculated as $\Delta \eta^{(m)}=2 \pi /\left(2 M_{2}+1\right)$ wherein

$$
\begin{aligned}
& M_{2}=\left\lfloor\chi_{2}^{\eta} M_{1}\right\rfloor+1, \\
& M_{1}=\left\lfloor 2 \chi_{1}^{\eta} W_{\eta}\right\rfloor+1 .
\end{aligned}
$$

The truncated interpolation which is enhanced by the Tschebyscheff or approximate prolate spheroidal wave functions is usually referred to as optimal sampling interpolation (OSI) [17].

Once the phase factor and sampling steps for the reduced monostatic field on a certain curve have been fully characterized, the method can be directly applied to arbitrary acquisition surfaces by using a double parametrization of the surface [2].

\section{Indirect holography}

The monostatic phaseless scattering depicted in Fig. 1 provides the following acquired hologram [15]:

$$
I(\eta)=\left|E_{s}^{(m)}(\eta)+E_{\text {ref }}(\eta)\right|^{2},
$$

wherein $E_{s}^{(m)}$ is the field scattered by the object and received by the Rx probe; and $E_{r e f}$ is the reference field that is synthetically generated by changing the phase of the reference field for each position of the antennas. Thus, the reference field is given by:

$$
E_{r e f}(\eta)=A e^{j \gamma(\eta)}
$$

with $A$ being the complex amplitude of the reference signal.

Considering the phase reduction factor for the monostatic field and applying the reasoning in [16], it is convenient to choose the phase of the reference field as:

$$
\gamma(\eta)=-2 \psi(\eta)-k_{\eta}^{p w} \eta
$$

wherein $k_{\eta}^{p w}$ is the wavenumber of a plane wave in the parametric domain that will be used later to retrieve the phase.

Inserting (10) and (9) into (8), the modified hologram [15], [14], [19] can be expressed as:

$$
\begin{aligned}
I^{\prime}(\eta) & =I(\eta)-A^{2}-\left|E_{s}^{(m)}(\eta)\right|^{2} \\
& =A F_{s}^{(m)}(\eta) e^{-j k_{\eta}^{p w} \eta}+A\left(F_{s}^{(m)}(\eta)\right)^{*} e^{j k_{\eta}^{p w} \eta},
\end{aligned}
$$

wherein the asterisk denotes complex conjugated and $F_{s}^{(m)}$ is the monostatic field $E_{s}^{(m)}$ reduced by the phase factor for monostatic field acquisition that has been determined to be equal to $2 \psi$ as previously shown. Thus, the Fourier transform of the modified hologram $\tilde{I}^{\prime}\left(k_{\eta}\right)=\mathcal{F}\left\{I^{\prime}(\eta)\right\}$ is given by:

$$
\tilde{I}^{\prime}\left(k_{\eta}\right)=A \tilde{F}_{s}^{(m)}\left(k_{\eta}-k_{\eta}^{p w}\right)+A\left(F_{s}^{(m)}\left(-k_{\eta}-k_{\eta}^{p w}\right)\right)^{*} \text {. }
$$

If the wave number is greater than the effective bandwidth of the reduced monostatic field, that is,

$$
k_{\eta}^{p w} \geqq 2 \chi_{1}^{\eta} W_{\eta},
$$

then, the two spectral components in (12) can be filtered out with a simple window in the spectral domain. If the first term is filtered out, then the reduced monostatic field can be retrieved by moving back this term to the parametric domain and dividing the result by the equivalent plane wave:

$$
E_{r e f}^{p w}(\eta)=A e^{-j k_{\eta}^{p w} \eta}
$$

\section{NUMERICAL RESULTS}

In this section, several method-of-moments-based numerical simulations are carried out to illustrate the previously described phase retrieval.

The scanning surface is a circle where a plane-polar acquisition is accomplished. This surface is parametrized in terms of the azimuthal angle $\phi$ and the polar radius $\rho$; this scheme enables the analytical evaluation of the bistatic spatial bandwidth as well as the phase factor for sources that can be enclosed by certain revolution geometries such as a disk 


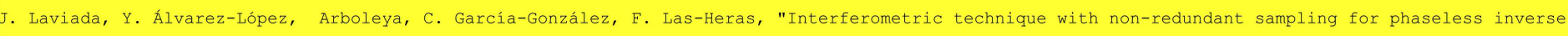
scattering" IEEE Transactions on Antennas and Propagation, vol. 62, no 2, pp. 223-230, 2014.

which is under IEEE copyright. You can check the final version in the following link

http: //ieeexplore. ieee.org/stamp/stamp.jsp?arnumber $=6690210$

or a sphere [2]. The parameter for the radial lines is denoted by a variable $\xi$ whereas the parameter for the azimuthal lines is directly the angle $\phi$ (see the discussion in [16] for further details about the parameter choice).

The phase shifting is only applied along the azimuthal angle as detailed in [16] so that an oversampling factor is not required for the azimuthal coordinate (see further details in [16]).

The common steps to solve each example are next described as follows:

1) The modified hologram is computed according to (8)(10).

2) The term $\tilde{F}_{s}^{(m)}\left(\xi_{u}, k_{\phi}-k_{\phi}^{p w}\right)$ is recovered from the spectral domain (12) by applying a window from 0 to $\pi / \Delta \eta$ for each azimuthal circus of radius $\rho\left(\xi_{u}\right)$.

3) The monostatic reduced field is retrieved by dividing the result from the previous step by the plane-wave in the parametric domain $E_{r e f}^{p w}=A e^{-j k_{\phi}^{p w} \phi}$.

4) The field is retrieved via interpolation on a rectangular grid of equally spaced points.

In all the results, the number of samples for the interpolation is set to $p=5$. The transmitter and receiver antennas, which are separated $15 \mathrm{~cm}$ along the $x$-axis, are modeled with $\cos ^{q}(\theta)$ radiation patterns [20].

\section{A. Single frequency plane-polar acquisition}

In this example, a metallic ellipse centered at the origin and lying in the $X Y$ plane is considered as OUT. The plate is perforated with the letters 'UO' (see Fig. 3a). This geometry can be enclosed by a disk of radius $a=6 \lambda$ and, therefore, this model is used to compute the parameterization of the azimuthal and meridian lines of the scanning surface as well as the bistatic phase factor according to [2]. However, since a more general model can be achieved by considering the sources enclosed by a sphere with the same radius, these results are also reported for the sake of completeness.

The Tx and Rx radiation patterns are modeled by setting $q=11$ which can be considered a reasonable model for conventional horns since it corresponds to a directivity equals to approximately $16.6 \mathrm{dBi}$. Both antennas are considered to be $y$-polarized. The working frequency is fixed at $f=15 \mathrm{GHz}$.

The distance from the object to the acquisition plane is $40 \mathrm{~cm}$. The observation domain is restricted to a maximum radius of $\rho_{\max }=20 \lambda$ that has been observed to be sufficiently large to acquire most of the radiated power.

After retrieving the phase, the field is computed on a square of edge $24 \lambda$ via OSI with sampling steps $\Delta x=\Delta y=\lambda / 4$ and, then, backpropagated to the aperture. The number of points in this square grid is 9409 .

Relative errors between the field retrieved in the square grid and the field are directly computed, and shown in Table I for several input parameter values. In this table, the factor $\left(\chi_{1}^{\phi}\right)^{\prime}$ is used instead of the real bandwidth enlargement factor $\chi_{1}^{\phi}$ because it does not depend on the radius of the acquisition circumference as discussed in [16]. The relative error is defined as:

$$
e=\frac{\left\|\hat{\bar{E}}_{s}^{(m)}-\bar{E}_{s}^{(m)}\right\|_{2}}{\left\|\bar{E}_{s}^{(m)}\right\|_{2}}
$$

wherein $\bar{E}_{s}^{(m)}$ is a vector containing the samples of the exact, that is, directly computed, scattered field; $\hat{\bar{E}}_{s}^{(m)}$ is the vector containing the samples of the field retrieved by OSI from the non-redundant set of samples; and $\|\cdot\|_{2}$ denotes Euclidean norm.

This table confirms that the bandwidth enlargement and oversampling factors should not be set to their minimum values to avoid high relative errors. Nevertheless, if the oversampling factor $\chi_{2}^{\xi}$ is increased from 1.0 to 1.2 , the relative error is drastically reduced with a relatively small increment of the number of acquisition points. Moreover, an increment of the bandwidth enlargement factor results in a relatively small error reduction if the oversampling factor has already been increased to 1.2. Hence, the choice $\chi_{1}^{\xi}=\left(\chi_{1}^{\phi}\right)^{\prime}=1.0$ and $\chi_{2}^{\xi}=1.2$ seem to provide a fair trade-off between relative error and the number of acquisition points.

In addition, Table I also confirms that considering that the sources can be enclosed by a disk is more efficient than the sphere model in terms of error value and number of sampling points.

Conventional sampling rules for plane-polar acquisition of bistatic field involve steps equal to $\Delta \rho^{(b)}=\lambda / 2$ and $\Delta \phi^{(b)}=\frac{\lambda}{2 \rho}$ [17]. Since the bandwidth of the monostatic field is doubled, then the sampling steps must be half of the previous ones, i.e., $\Delta \rho^{(m)}=\lambda / 4$ and $\Delta \phi^{(m)}=\frac{\lambda}{4 \rho}$. Finally, if the phase shifting is applied along $\phi$ and the minimum value of $k_{\phi}^{p w}$ given in (13) is considered, then the bandwidth along $\phi$ is again doubled so that the sampling steps for Leith-Upatnieks holography become $\Delta \rho=\lambda / 4$ and $\Delta \phi=\frac{\lambda}{8 \rho}$. Thus, the number of acquisition points would be 40756 .

Table I

RESULTS FOR THE SIMULATED UO LETTERS ENCLOSED BY A METALLIC ELLIPSE.

\begin{tabular}{|c|c|c|c|c|c|}
\hline & \multicolumn{3}{|c|}{ Inputs } & \multicolumn{2}{c|}{ Outputs } \\
\hline \hline Source model & $\chi_{1}^{\xi}$ & $\chi_{2}^{\xi}$ & $\left(\chi_{1}^{\phi}\right)^{\prime}$ & $\begin{array}{c}\text { Sample } \\
\text { points } \\
\text { (plane-polar } \\
\text { grid) }\end{array}$ & $\begin{array}{c}\text { Error } \\
\text { (square } \\
\text { grid) }\end{array}$ \\
\hline Regular sampling & - & - & - & 40756 & - \\
\hline Sphere & 1.0 & 1.0 & 1.0 & 2517 & $8.86 \%$ \\
\hline Sphere & 1.0 & 1.2 & 1.0 & 3013 & $1.82 \%$ \\
\hline Sphere & 1.2 & 1.2 & 1.2 & 4603 & $1.5 \%$ \\
\hline Disk & 1.0 & 1.0 & 1.0 & 2111 & $7.72 \%$ \\
\hline Disk & 1.0 & 1.2 & 1.0 & 2442 & $1.68 \%$ \\
\hline Disk & 1.2 & 1.2 & 1.2 & 3906 & $1.5 \%$ \\
\hline
\end{tabular}

Fig. 3 shows that the aforementioned errors do not result in any artifacts in the retrieved reflectivity, which has an excellent agreement with the one calculated from the directly computed field. The reflectivity has been efficiently computed by means of 2D fast fourier transforms (FFTs) as detailed in [21]. 


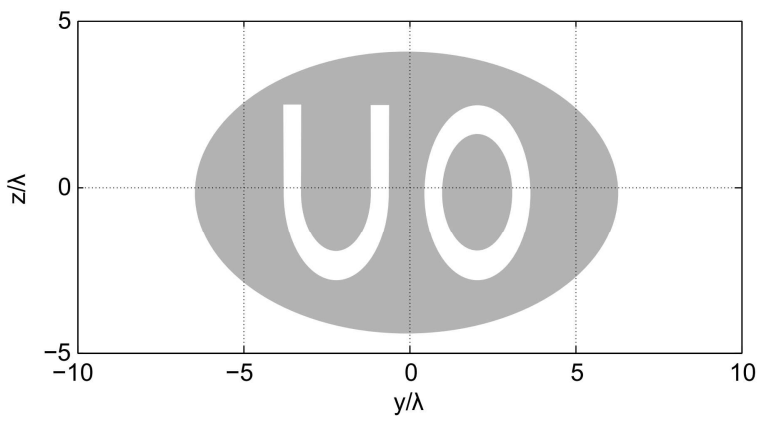

(a) Geometry for the 'UO' letters.

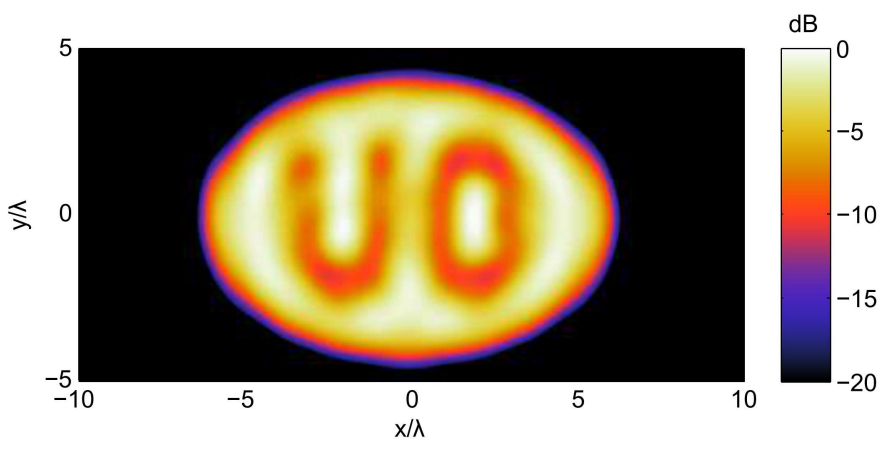

(b) Backpropagation from the exact field.

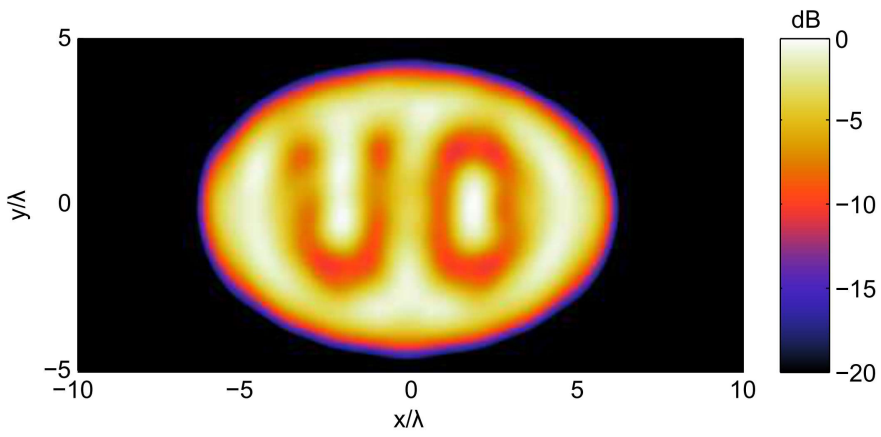

(c) Backpropagation from the retrieved field for $\chi_{1}^{\xi}=\left(\chi_{1}^{\phi}\right)^{\prime}=1.0$ and $\chi_{2}^{\xi}=1.2$ with sources enclosed by a disk.

Figure 3. Normalized reflectivity for the metallic ellipse with the UO letters.

\section{B. Multifrequency plane-polar acquisition}

In the next example, a multifrequency acquisition is accomplished by carrying out a frequency sweep from $12 \mathrm{GHz}$ to $18 \mathrm{GHz}$ with a frequency step equals to $\Delta f=250 \mathrm{MHz}$. Transmitter and receiver antennas are modeled with $q=1$ which can be considered a fair model for open ended waveguides. Under this model, the approximate directivity for both antennas, which are $x$-polarized, is $7.8 \mathrm{dBi}$.

According to the results in the previous section, the bandwidth enlargement factors are set to $\chi_{1}^{\xi}=\left(\chi_{1}^{\phi}\right)^{\prime}=1.0$ and the oversampling factor is set to $\chi_{2}^{\xi}=1.2$.

The OUT is the isosceles trapezoid with a hole shown in Fig. 4. The object has been rotated $45^{\circ}$ around the $x$-axis.

The scanning plane is set at $z=1 \mathrm{~m}$ and the maximum radius is limited to $\rho_{\max }=1.4 \mathrm{~m}$. The acquisition points are considered to be at the highest frequency resulting in 4888 points. Conventional sampling approaches for this acquisition radius would result in 703,193 points.

It is important to notice that the spatial bandwidth for the parametric domain is computed by considering that the object is enclosed by an sphere of radius $a=11 \mathrm{~cm}$ instead of a disk. It is done so that the acquisition circles can be considered azimuthal circumferences in order to apply the expressions in [2].

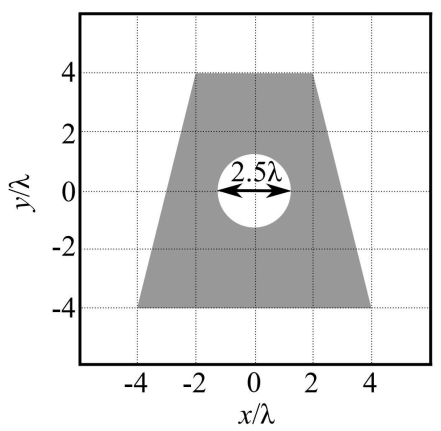

Figure 4. Geometry of the trapezoid with hole before rotating around the $x$-axis. The electrical dimensions are referred to the wavelength at the central frequency $f=15 \mathrm{GHz}$.

Once the field has been acquired, it is retrieved on a rectangle at the scanning plane, that is, $z=1 \mathrm{~m}$, spanning from $x=-35 \mathrm{~cm}$ to $x=35 \mathrm{~cm}$ and from $y=-60 \mathrm{~cm}$ to $y=135 \mathrm{~cm}$. The step between the points is set to $\Delta x=\Delta y=\lambda_{\min } / 4$ wherein $\lambda_{\min }$ is the wavelength at the maximum frequency. The number of points in this acquisition window is 79261 .

The phase shifting is applied along the azimuthal coordinate $\phi$ by means of the mechanical phase shifting detailed in [11]. The error in the retrieved field ranges from $0.62 \%$ to $1.48 \%$. Therefore, the agreement in the retrieved profile is expected to be very good. In general, the error is lower at the lowest frequencies wherein the sampling is denser than the minimum one. On the other hand, the error increases at the highest frequencies wherein the sampling is close to the minimum one.

The object retrieved from the field values in the rectangular window by means of the algorithm detailed in [22] is shown in Fig. 5 with an excellent agreement with the OUT.

\section{MEAsurements Results}

Finally, experimental validation of the proposed technique is presented. A planar measurement setup, shown in Fig. 6, is used. The phase shifting has been accomplished again by applying the mechanical displacements detailed in [11] instead of using a phase shifter. All the measurements have been accomplished at $18 \mathrm{GHz}$ by acquiring the field on a plane-polar surface with a maximum radius $\rho_{\max }=16 \mathrm{~cm}$. Several OUTs have been considered for this example (see geometries in Fig. 7a, Fig. 7c and Fig. 7e). These objects have been constructed from square brass plates of edge $10 \mathrm{~cm}$ so that the maximum radius of the disk enclosing the OUTs is $a=5 \sqrt{2} \mathrm{~cm}$. The transmitter and receiver antennas are two horn antennas separated by $23.5 \mathrm{~cm}$. The distance from the plane containing the aperture of the antennas to the OUT plane is $50 \mathrm{~cm}$. 


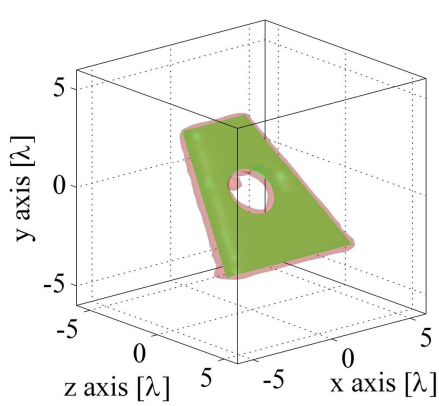

(a) $3 \mathrm{D}$ view

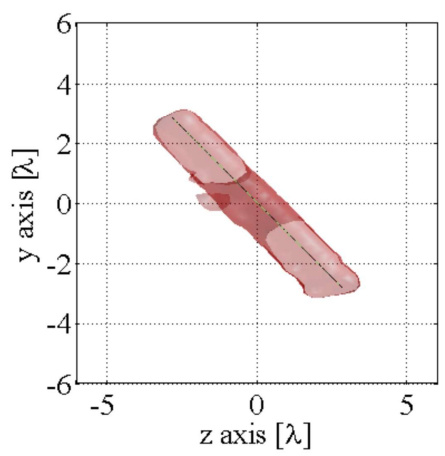

(c) YZ plane

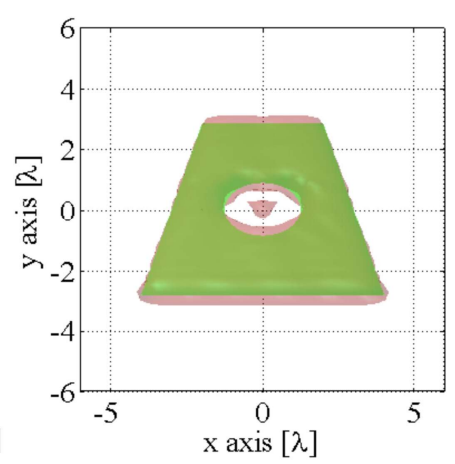

(b) XY plane

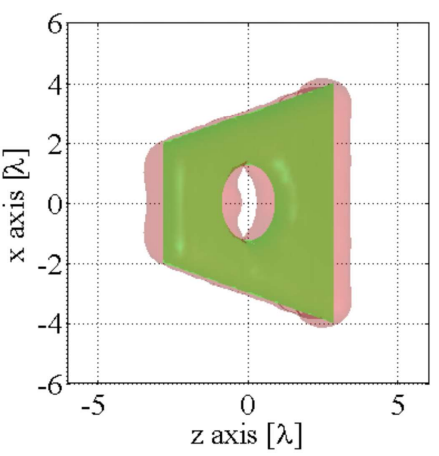

(d) XZ plane
Figure 5. Isosurfaces at $-12 \mathrm{~dB}$ (see [23], [22]) for the trapezoid geometry. The electrical dimensions are referred to the wavelength at the central frequency $f=15 \mathrm{GHz}$.

The bandwidth enlargement and oversampling factors and the number of samples for the interpolation have been set as in the previous example. Taking into consideration the described setup, the resulting number of acquisition points is 324. A conventional sampling for a monostatic plane-polar acquisition with the same radius would result into 9331 points achieving a 28 times reduction in the number of samples.

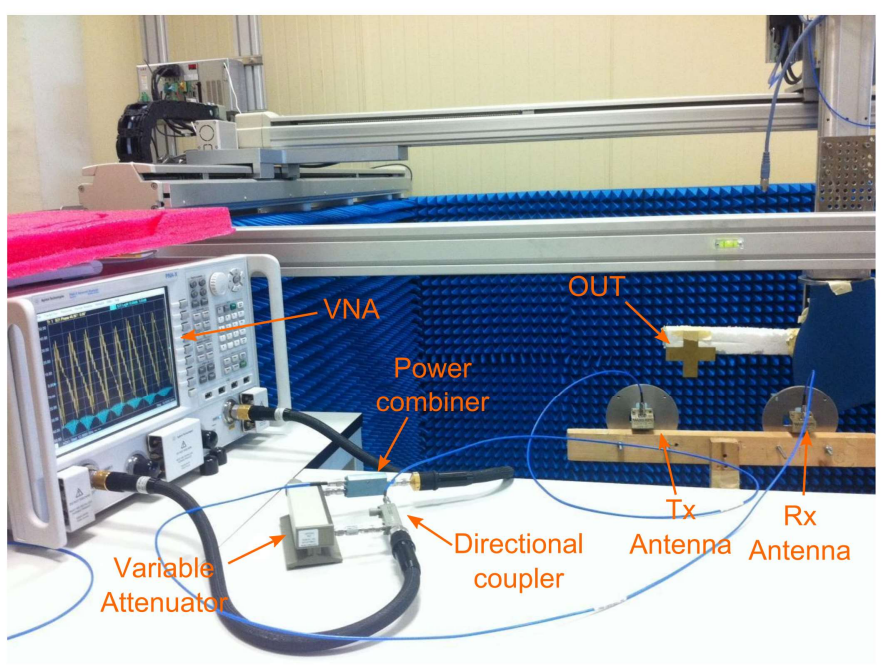

Figure 6. Implementation of the setup at $18 \mathrm{GHz}$.

In order to measure the amplitude, a vector network analyzer (VNA) has been used to acquire the $S_{21}$ parameter. Only the square of the amplitude is stored so that the phase information is discarded. In a final system, this measurement would be accomplished by a power-meter.

The steps that have been followed for each OUT are:

1) The OUT is moved along the acquisition points so that the hologram in (8) is acquired.

2) The reference branch is unplugged and the $50 \Omega$ loads are placed at the open ends. The OUT is moved again along the acquisition points so that $\left|E_{s}^{(m)}\right|^{2}$ is acquired.

3) The modified hologram for the reduced field is computed and the data is recovered by filtering the spectra as previously detailed.

4) The reduced field is retrieved by means of OSI on a square grid whose edges are equal to $2 \rho_{\max }$. The sampling step is $\Delta x=\Delta y=\lambda / 4$.

5) The field is recovered from the reduced field and then the reflectivity is computed as in the first numerical example by means of FFTs [21]. . A zero padding is used to improve the rendering of the retrieved profile.

The error as defined in the first numerical example is $16 \%$, $12 \%$ and $25 \%$ for the geometries shown in Fig. 7a, 7c and $7 \mathrm{e}$, respectively. This error is computed by comparing the field retrieved in the square grid with the one measured in the step \#2. In addition, the phase shift, whose associate errors have been quantified in [11], corresponding to the phase term $\psi$ is computationally removed. These errors are higher than in the previous example due to the multiple factors including measurement noise, mechanical positioning accuracy and phase shifting error.

The retrieved reflectivity for the different geometries is shown in Fig. 7b, Fig. 7d and Fig. 7f. Despite the reduced number of points, the profile of the objects is still clearly visible with a resolution close to the wavelength.

\section{CONCLUSiOnS}

A phaseless monostatic planar setup has been presented in this paper. In comparison with some of the already available techniques (e.g., [3], [4], [5], [6], [7]), the proposed monostatic setup involves less information since only one acquisition point is used for each incident field. However, experimental results available in the literature reveals that this planar monostatic acquisition can provide valuable information for defense and security purposes (e.g., [18], [21]).

In this setup, Leith-Upatnieks holography has been applied to acquire the scattered field and, consequently, it is iterationfree as opposed to other phase retrieval schemes [3], [4]. Thus, any potential stagnation problem is avoided. In addition, it does not require any specific geometry restrictions to avoid local minima [4], [5]. In return, it requires an accurate characterization of the reference wave as well as some extra equipment. However, these drawbacks have been alleviated by applying recent advances in the field (see [11]) .

The scanning system herein presented exhibits the following characteristics: i) low cost due to the amplitude-only acquisition; ii) efficient acquisition due to the very reduced number of acquisition points; iii) computationally inexpensive post- 


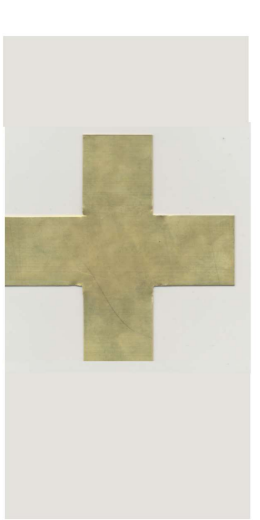

(a)

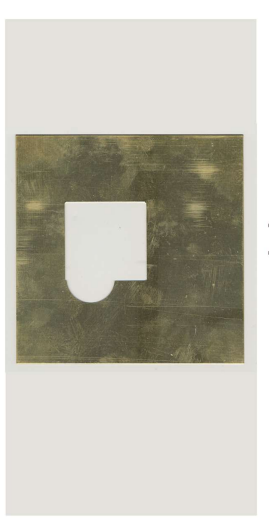

(c)

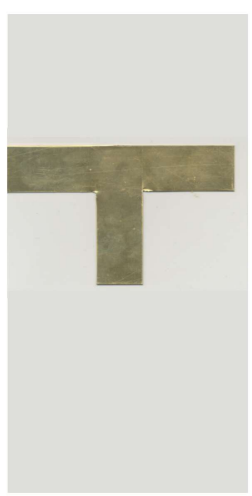

(e)

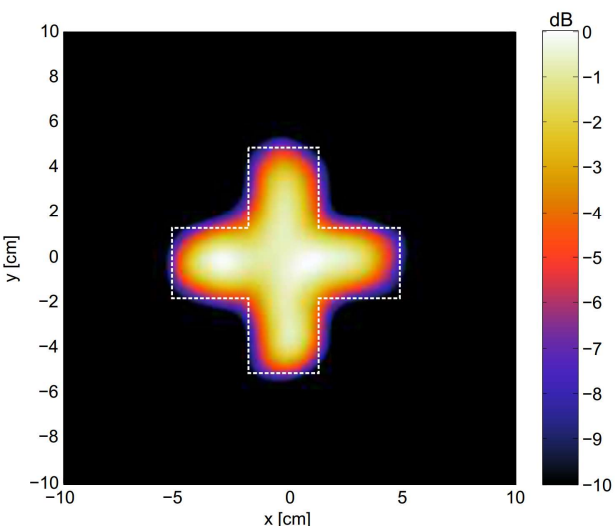

(b)

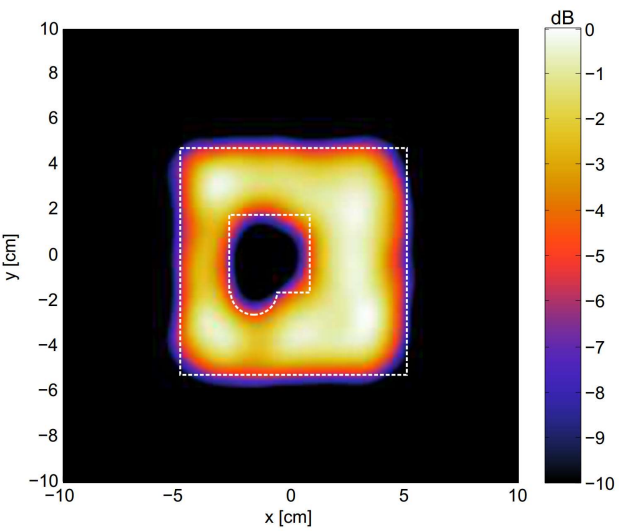

(d)

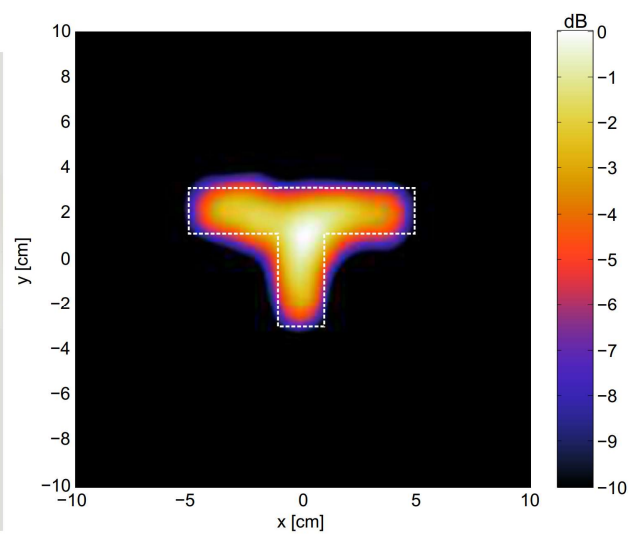

(f)
Figure 7. Geometries of the OUTs and normalized reflectivity.

processing that enables the evaluation of the field everywhere inside the acquisition surface.

Despite the aforementioned advantages, accuracy has not suffered a significant degradation with respect to a fullwave acquisition as it has been proved for several numerical examples. In addition, the practical implementation has been validated with multiple examples at microwave frequencies.

\section{REFERENCES}

[1] O. M. Bucci and T. Isernia, "Electromagnetic inverse scattering: Retrievable information and measurement strategies," Radio Science, vol. 32, no. 6, pp. 2123-2137, november-december 1997.
[2] O. M. Bucci, C. Gennarelli, and C. Savarese, "Representation of electromagnetic fields over arbitrary surfaces by a finite and nonredundant number of samples," IEEE Trans. Antennas Propagat., vol. 46, no. 3, pp. 351-359, Mar. 1998.

[3] L. Crocco, M. D'Urso, and T. Isernia, "Inverse scattering from phaseless measurements of the total field on a closed curve," J. Opt. Soc. Am. A, vol. 21, no. 4, pp. 622-631, Apr. 2004.

[4] O. M. Bucci, L. Crocco, M. D'Urso, and T. Isernia, "Inverse scattering from phaseless measurements of the total field on open lines," Journal of Optical Society of America, vol. 23, no. 10, pp. 2566-2577, Oct. 2006.

[5] M. D'Urso, K. Belkebir, L. Crocco, T. Isernia, and A. Litman, "Phaseless imaging with experimental data: facts and challenges," Journal of Optical Society of America, vol. 25, no. 1, pp. 271-281, Jan. 2008.

[6] L. Crocco, M. D'Urso, and T. Isernia, "Faithful phaseless nonlinear imaging from only-amplitude measurements of incident and total field," Optics Express, vol. 15, no. 7, pp. 3804-3815, Apr. 2007.

[7] $\_$, "Quantitative imaging from diffracted fields intensities: an inversion method and its experimental validation," Journal of Modern Optics, vol. 57, no. 9, pp. 777-782, Jan. 2010 .

[8] E. N. Leith and J. Upatnieks, "Reconstructed wavefronts and communication theory," J. Opt. Soc. Amer., vol. 52, pp. 1123-1128, 1962.

[9] G. Junkin, T. Huang, and J. Bennett, "Holographic testing of terahertz antennas," IEEE Trans. Antennas Propagat., vol. 48, no. 3, pp. 409 -417, Mar. 2000.

[10] A. Tamminen, J. Ala-Laurinaho, and A. V. Räisänen, "Imaging with indirect holographic method at $310 \mathrm{GHz}$," in Proc. XXXI Finnish URSI convention on radio science and electromagnetics, 2008, pp. 31-32.

[11] J. Laviada, Y. Álvarez López, C. García-González, A. Arboleya, and F. Las-Heras, "A modified phaseless inverse scattering setup based on indirect holography implemented at submillimeter-wave band," IEEE Trans. Antennas Propagat., vol. 61, no. 9, pp. 4876-4881, Sep. 2013.

[12] J. Laviada, Y. Álvarez López C. García-González, C. Vázquez-Antuña, S. Ver-Hoeye, M. Fernández-García, G. Hotopan, R. Camblor, and F. Las-Heras, "A novel phaseless frequency scanning based on indirect holography," Journal of Electromagnetic Waves and Applications, vol. 27, no. 4, pp. 275-296, 2013.

[13] D. Smith, M. Leach, and A. Sambell, "Microwave indirect holographic imaging using an adaptation of optical techniques," IEEE Microwave and Wireless Component Letters, vol. 13, no. 9, pp. 379-381, Sep. 2003.

[14] V. Schejbal, V. Kovarik, and D. Cermak, "Synthesized-reference-wave holography for determining antenna radiation characteristics," IEEE Antennas Propagat. Mag., vol. 50, no. 5, pp. 71 - 83, Oct. 2008.

[15] J. Laviada, Y. Âlvarez López, C. García-González, A. Arboleya, and F. Las-Heras, "Inverse scattering with phase retrieval based on indirect holography via synthetic plane-waves," IET Microwave, Antennas and Propagation, vol. 6, no. 12, pp. 1389-1398, 2012.

[16] J. Laviada and F. Las-Heras, "Phaseless antenna measurement on nonredundant sample points via leith-upatnieks holography," IEEE Transactions on Antennas and Propagation, vol. 61, no. 8, pp. 4036-4044, Aug. 2013.

[17] O. Bucci, C. Gennarelli, and C. Savarese, "Fast and accurate nearfield far-field transformation by sampling interpolation of plane polar measurements," IEEE Trans. Antennas Propagat., vol. 39, pp. 48-55, Jan. 1991.

[18] S. Ahmed, A. Schiessl, F. Gumbmann, M. Tiebout, S. Methfessel, and L. Schmidt, "Advanced microwave imaging," IEEE Microwave Magazine, vol. 13, no. 6, pp. 26-43, 2012.

[19] D. Smith, M. Leach, M. Elsdon, and S. J. Foti, "Indirect holographic techniques for determining antenna radiation characteristics and imaging aperture fields," IEEE Antennas Propagat. Mag., vol. 49, no. 1, pp. 54 -67, Feb. 2007.

[20] Y. T. Lo and S. W. Lee, Eds., Antenna Handbook: Antenna Fundamentals and Mathematical Techniques. New York: Van Nostrand Reinhold: Chapman \& Hall, 1993.

[21] D. M. Sheen, D. L. McMakin, , and T. E. Hall, "Three-dimensional millimeter-wave imaging for concealed weapon detection," IEEE Transactions on Microwave Theory and Techniques, vol. 49, no. 9, pp. 15811592, Sep. 2001.

[22] Y. Alvarez, J. Laviada, L. Tirado, C. Garcia, J. Martinez, F. LasHeras, and C. Rappaport, "Inverse fast multipole method for monostatic imaging applications," IEEE Geoscience and Remote Sensing Letters, vol. 10, no. 5, pp. 1239-1243, 2013.

[23] Y. Álvarez, J. A. Martínez, F. Las-Heras, and C. Rappaport, "An inverse fast multipole method for geometry reconstruction using scattered field information," IEEE Trans. Antennas Propagat., vol. 60, no. 7, pp. 33513360, Jul. 2012. 


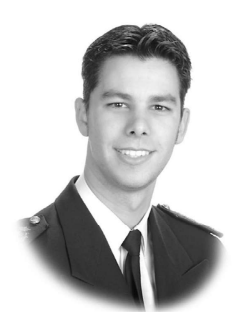

Jaime Laviada was born in Gijón, Spain. He received the M.S. degree in telecommunication engineering and the Ph.D. degree from the University of Oviedo, Gijón, Spain, in 2005 and 2010, respectively.

In 2006, he joined the research group Signal Theory and Communications, Department of Electrical Engineering, University of Oviedo. He was a Visiting Scholar in the Electromagnetics and Communications Lab, Pennsylvania State University, State College, PA, USA, during 2007 and 2008.

He has been involved in multiple national and European projects as well as multinational company contracts. His main research interests are in numerical efficient techniques applied to antenna measurements, method of moments, and antenna pattern synthesis.

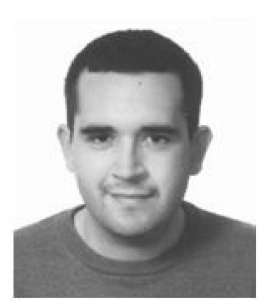

Yuri Alvarez (S'06-M'09) was born in Langreo, Spain, in 1983. He received the M.S. and Ph.D. degrees in telecommunication engineering in 2006 and 2009, respectively.

He was a Visiting Scholar in the Department of Electrical Engineering and Computer Science, Syracuse University, Syracuse, NY, USA, in 2006 and 2008; and a Visiting Postdoc at the Gordon Center for Subsurface Sensing and Imaging Systems (CenSSIS)_ALERT Center of Excellence, Northeastern University, Boston, MA, USA, in 2011, 2012, and 2013. He is currently an Assistant Professor at the Signal Theory and Communications of the University of Oviedo, Gijón, Spain.

Dr. Alvarez received the 2011 Regional and National Awards to the Best Ph.D. Thesis on Telecommunication Engineering (category: security and defense). His interests and research studies have been focused on antenna diagnostics, antenna measurement techniques, RF techniques for indoor location, and inverse scattering and imaging techniques.

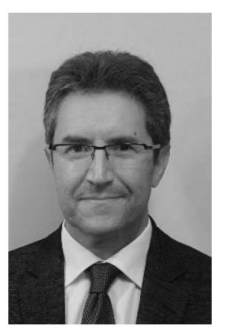

Fernando Las-Heras (M'86, SM'08) received the M.S. degree in 1987 and the Ph.D. degree in 1990, both in Telecommunication Engineering, from the Technical University of Madrid (UPM). From 1988 to 1990 , he was a National Graduate Research Fellow. From 1991 to 2000 he held a position of Associate Professor at the Department of Signal, Systems and Radiocommunications of the UPM. From December 2003 he holds a Full-Professor position at the University of Oviedo where he has been Vice-dean for Telecommunication Engineering at the Technical School of Engineering at Gijón from 2004 to 2008. From 2001 he heads the research group Signal Theory and Communications TSCUNIOVI at the Dept. of Electrical Engineering of the University of Oviedo. He was Visiting Lecturer at the National University of Engineering in Peru in 1996, Visiting Researcher at Syracuse University, New York, in 2000, and short term Visiting Lecturer at ESIGELEC in France since 2005. From 2005 he holds the Telefónica Chair ICTs applied to Environment and Climate Change in the University of Oviedo and from 2010 he is member of the Science, Technology and Innovation Council of Asturias, Spain. He has authored over 300 technical journal and conference papers mainly in the areas of antenna design and the inverse electromagnetic problem with applications in diagnostic, measurement and synthesis of antennas, phaseless techniques, propagation, and microwave to $\mathrm{THz}$ imaging and localization.

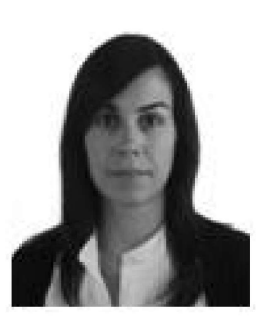

Ana Arboleya Arboleya received de M.Sc. degree in telecommunication engineering from the University of Oviedo, Spain, in 2009, where she is currently working toward the Ph.D. degree. Since 2008, she has been working as a Research Assistant with the Signal Theory and Communications Area at the University of Oviedo. Her major research interests comprise antenna and RCS measurement systems and high frequency scattering measurement techniques.

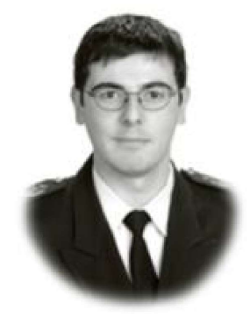

Cebrián García González (S'10) was born in Pola de Laviana (Spain) in 1983. He received the M.Sc. degree in telecommunication engineering from the University of Oviedo, Gijón, Spain, in 2010, where he is currently working toward the Ph.D. degree. Since 2010, he has been working as a Research Assistant with the Signal Theory and Communications Area, University of Oviedo. His research efforts are focused on the reconstruction of electromagnetic sources from field measurements, inverse scattering problem and imaging techniques. 